\title{
Calcium pyrophosphate dihydrate deposition in the intervertebral discs in a case of Wilson's disease
}

\author{
J McCLURE, PS SMITH
}

From the Division of Tissue Pathology, Institute of Medical and Veterinary Science, Frome Road, Adelaide, South Australia 5000

SUMMARY The vertebral column from a known case of Wilson's disease (hepatolenticular degeneration) was examined by radiological, histological, histochemical and $\boldsymbol{x}$-ray microanalytical techniques which demonstrated the presence of focal depositions of calcium pyrophosphate dihydrate (CPPD) in the intervertebral discs. These deposits were present in both the annulus fibrosus and the nucleus pulposus but in certain discs the deposits were concentrated near the interface between disc and vertebral body bone endplates. At these sites there was new bone formation with narrowing of the discs, irregularity and sclerosis of the bone endplates and exostosis.

As Hamilton ${ }^{1}$ has pointed out chondrocalcinosis has been reported on two occasions ${ }^{23}$ in association with Wilson's disease (hepatolenticular degeneration). However, the precise chemical nature of the observed radiological calcifications is unknown.

The vertebral column from a case of Wilson's disease was made available after postmortem examination of the brain and spinal cord. The specimen was studied by various techniques to determine the presence of calcific deposits in the intervertebral discs and the nature of any attendant arthropathic effects.

\section{Case report}

The patient was a 40-year-old man who first presented 17 years prior to death with haemoptysis and was found to have moderate thrombocytopenia and splenomegaly. One year later he had an episode of haematemesis and melaena from a small duodenal ulcer. Splenectomy was performed and hepatic cirrhosis was noted at operation. Two years later a diagnosis of Wilson's disease was made. At that time there were stigmata of chronic liver disease, Kayser-Fleischer rings and a coarse intention tremor of the right hand. Treatment with penicillamine produced a tenfold increase in urinary copper excretion. His terminal illness was precipitated by haematemesis from oesophageal varices. He died in fulminant hepatic failure.

At postmortem examination there was a macronodular cirrhosis complicated by massive hepatic

Accepted for publication 19 January 1983 necrosis probably due to hypotension consequent to haematemesis. Estimations of copper in liver and kidney on a dry basis gave values of $21.7 \mathrm{mmol} / \mathrm{kg}$ (reference values $0.8-2.7 \mathrm{mmol} / \mathrm{kg}$ ) and 12.4 $\mathrm{mmol} / \mathrm{kg}$ (reference value $0 \cdot 2 \mathrm{mmol} / \mathrm{kg}$ ). The reference values are from Delves. ${ }^{4}$

\section{Material and methods}

A strip of vertebral column extending from the cervical to the lumbosacral region was obtained by cutting in anteroposterior plane to the right and left of the midline. The strip was $10 \mathrm{~mm}$ thick. It was $x$-rayed in a Faxitron cabinet $x$-ray machine. Portions of the intervertebral discs were excised for histological, histochemical, scanning electron microscopical and $x$-ray microanalytical examination. Sections were stained by haematoxylin and eosin, the von Kossa technique (with haematoxylin and eosin and the van Gieson counter-stains), alizarin red S and the morin technique. Unstained sections were mounted without coverslips and without removal of paraffin wax for examination by compensated polarising light microscopy. Blocks of vertebral body and attached intervertebral disc were embedded in Araldite and thin $(7 \mu \mathrm{m})$ sections cut without prior decalcification. These sections were also stained by the methods stated above and by the rhodanine method for the demonstration of copper.

Fragments of discs and adjacent vertebral bodies were fixed in glutaraldehyde, post fixed in osmium tetroxide, dehydrated in ethanol and embedded in Spurr's resin. Survey sections $(0.5 \mu \mathrm{m})$ were stained 


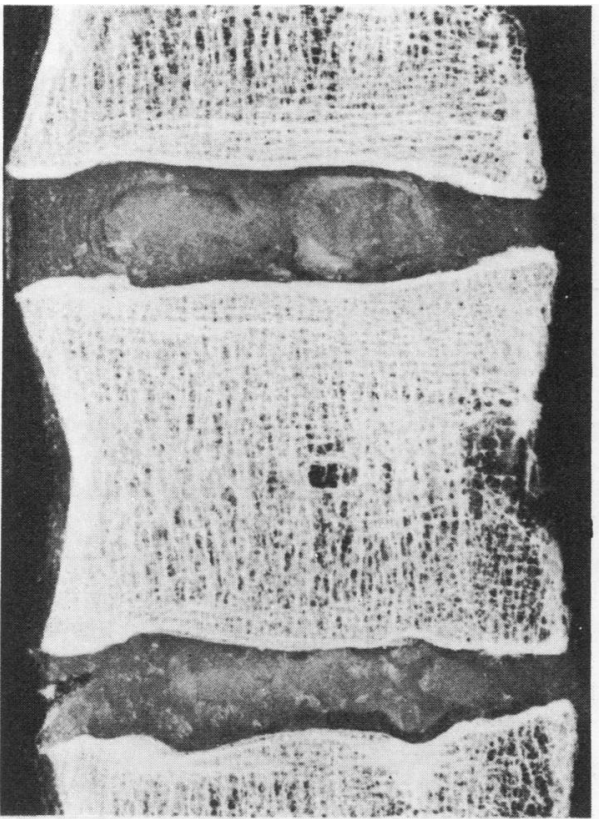

Fig. 1 Contact radiograph of the vertebral strip (anterior surface to the left). The small radio-opacities in the disc are crystalline deposits of CPPD.

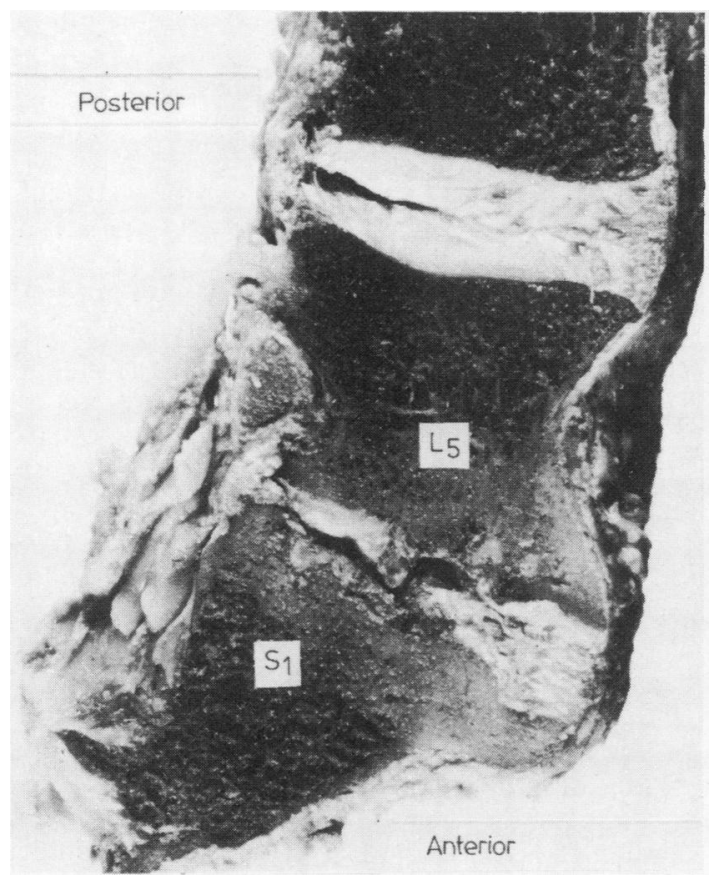

Fig. 2 The narrowing of the disc between L5 and S1 is shown. There is prominent anterior exostosis, sclerosis and irregularity of the bone-endplates.

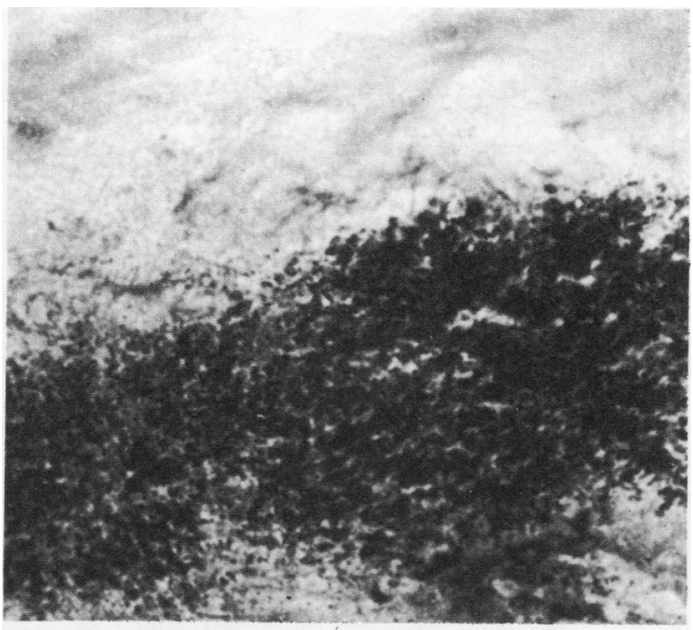

Fig. 3 Crystalline deposit in the nucleus pulposus. von Kossa $\times 400$

with toluidine blue, assessed and suitable blocks carbon coated (polishing was not required) for micro-analysis by a Jeol 733 superprobe model electron micro-analyser with a Kevex 7100 series energy dispersive spectrometer (accelerating voltage $15 \mathrm{kV}$, beam current $9 \mathrm{nA}$, spectrum collection time $20 \mathrm{~s}$, spectrometer take-off angle $=45^{\circ}$ ).

\section{PATHOLOGICAL FINDINGS}

Radiographs of the vertebral strip revealed numerous small (approximately $1 \mathrm{~mm}$ diameter) radioopacities randomly distributed in the intervertebral discs, in both the annulus and the nucleus (Fig. 1), and in the anterior spinal ligament. In this latter they were visible to the naked eye as discrete white deposits. Macroscopically and radiologically there was marked narrowing of the disc between the fifth lumbar (L5) and first sacral (S1) vertebrae with a prominent anterior exostosis, irregularity of the bone endplate and a dense sclerosis of the subjacent bone (Fig. 2). A similar process of lesser extent involved the disc between the third and fourth cervical vertebrae.

Microscopically the intervertebral disc opacities were discrete rounded masses (Fig. 3) which were basophilic on haematoxylin and eosin staining, von Kossa and alizarin red $S$ positive and exhibited green-white fluorescence after the application of the morin technique. Compensated polarising microscopy showed a weak positive birefringence. The deposits were present in both annulus and nucleus.

In the Araldite-embedded undecalcified section of vertebral bodies and intervening discs von Kossa and alizarin red $\mathrm{S}$ positive deposits were present in 


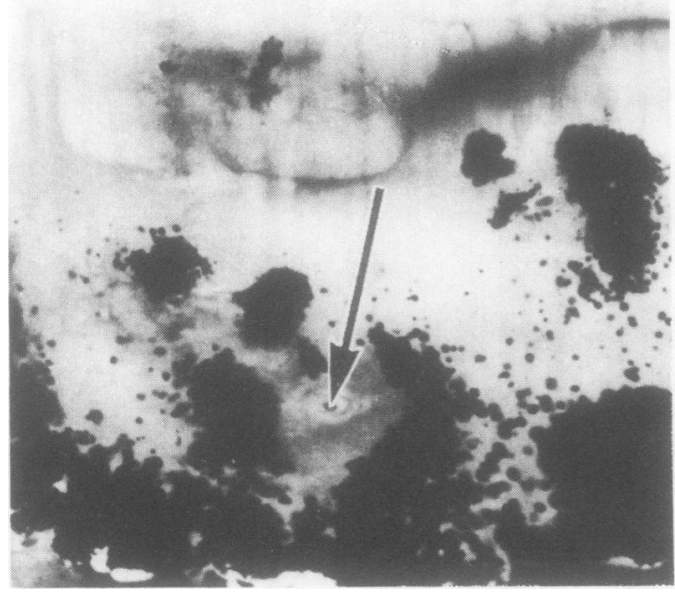

Fig. 4 Calcific deposits near and around chondrocytes (one is arrowed) in the cartilaginous endplates near the disc/bone interface. von Kossa with haematoxylin and eosin $\times 200$

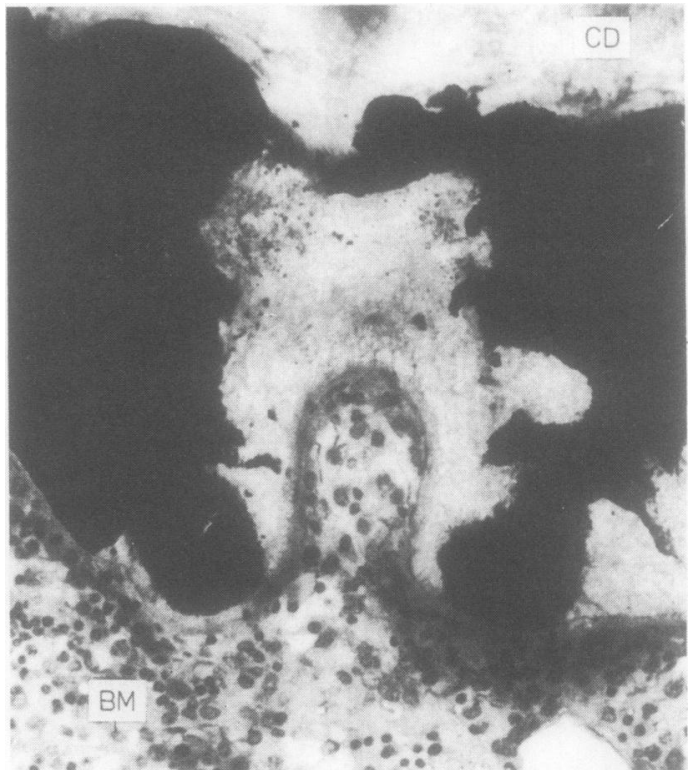

Fig. 5 New trabecular bone formation (stained black) producing irregularity of the disc/vertebral body interface. $C D$ is the cartilaginous endplate of the disc and $B M$ is the bone marrow of the vertebral body. von Kossa with haematoxylin and eosin $\times 200$

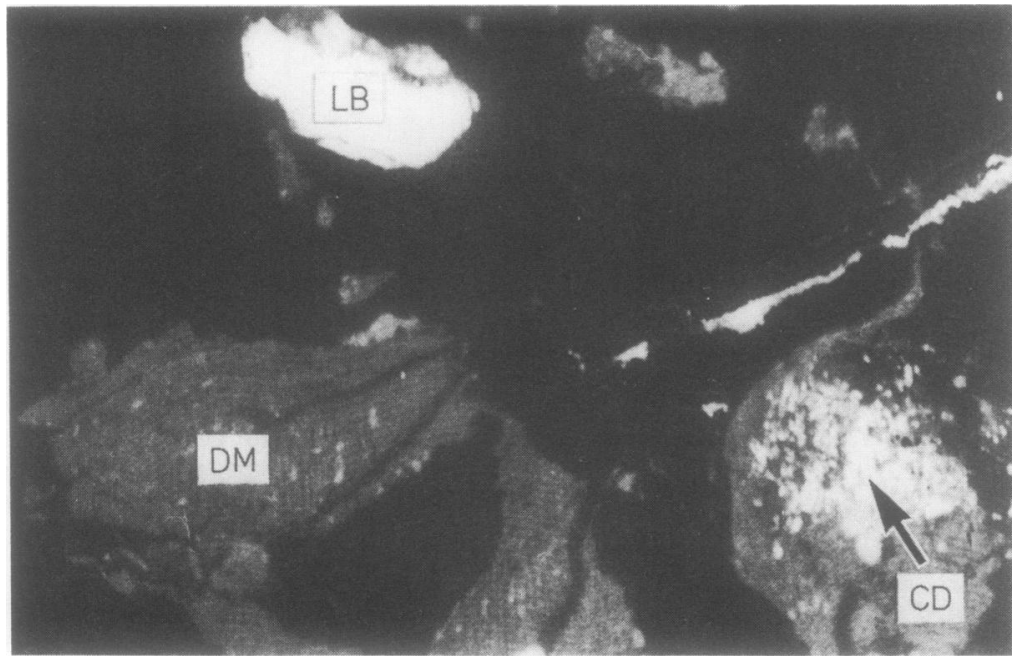

Fig. 6 Back scattered electron image of intervertebral disc material. $L B$ is lamellar bone, $D M$ is disc matrix and $C D$ is a crystalline deposit of $C P P D \times 170$

the matrix and around chondrocytes near the disc/ bone interface (Fig. 4). New trabecular bone formation in the endplate produced irregularity of the line of the interface and this effect was particularly marked in the disc between L5 and S1 (Fig. 5). Additionally there was marked peripheral exostosis.

Generally in the undecalcified sections there was no evidence of metabolic bone disease and specifically there was no evidence of osteomalacia.
The histochemical stains for copper were negative.

With the Jeol 733 superprobe the specimens were examined using back scattered electron imaging and three components in the mounts were distinguished ${ }_{0}^{-}$ by the relative brightness of their images. These $\overrightarrow{\mathbb{D}}$ were (i) rounded crystalline deposits (ii) disc matrix $\frac{?}{\mathbb{1}}$ (iii) portions of lamellar bone (Figs. 6 and 7); $x$-ray $\varrho$ spectra were obtained from two portions of lamellar bone and eight areas of crystalline deposition (Fig. 8 


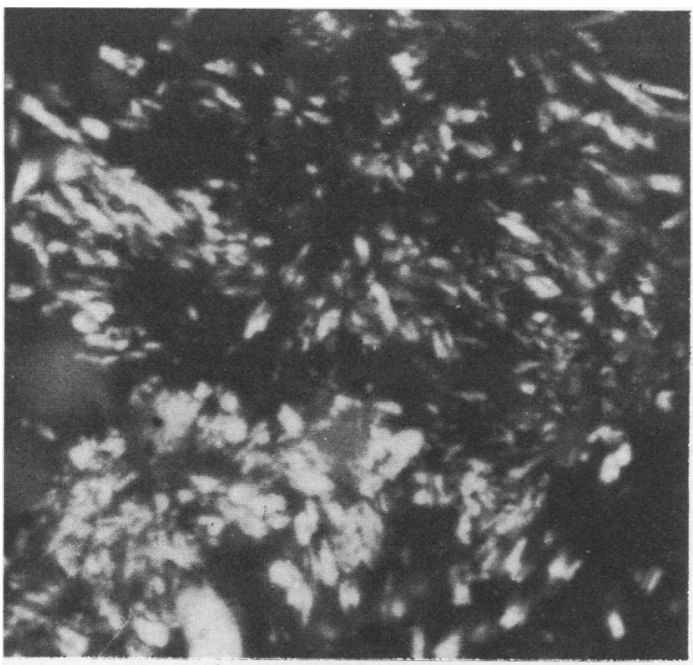

Fig. 7 Back scattered electron image of a crystalline deposit $\times 1650$

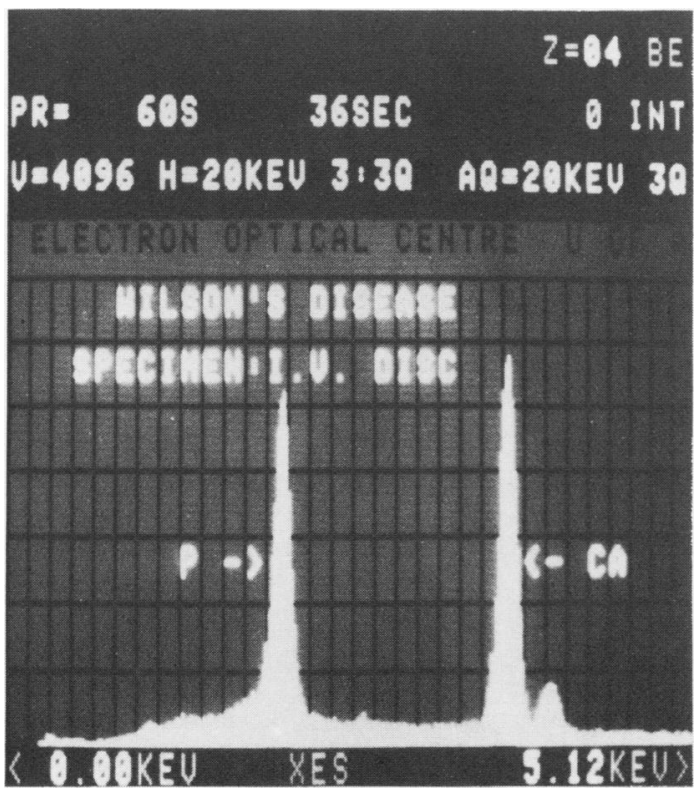

Fig. $8 x$-ray spectrum of a crystalline deposit showing the presence of phosphorus (P) and calcium (CA). The P:CA ratio is 0.96 .

8). Calcium (Ca) and phosphorus $(\mathrm{P})$ were present in both the crystalline deposits and the lamellar bone but in different proportions. The $x$-ray emissions for each area were measured and the ratio of $P: C a$ calculated. The mean value of this ratio for the lamellar bone was 0.57 and for the crystalline deposits it was $0 \cdot 96$.

Accumulations of copper were not detected in disc material or bone by $x$-ray microanalysis.

\section{Discussion}

Calcification of articular cartilage, joint capsules and tendinous insertions detected by radiological examination in a case of Wilson's disease was first described by Boudin et al. ${ }^{2}$ Feller and Schumacher ${ }^{3}$ reviewed 17 cases of known Wilson's disease and found skeletal abnormalities in five patients who were over 21 years of age. Multiple calcified loose bodies at the wrist were present in four, premature degenerative arthritis at the knees with chondromalacia patellae in four and chondrocalcinosis at the knees in two. The precise chemical nature of the calcific deposits was not elucidated in these cases. However, Feller and Schumacher concluded that the radio-opacities were due to deposition of calcium pyrophosphate dihydrate crystals (CPPD). Moreover, in vitro inhibition of erythrocyte pyrophosphatase activity in cupric and ferrous ions has been reported by McCartey et al $^{5}$ thereby suggesting a possible mechanism for pyrophosphate excess and deposition of calcium pyrophosphate both in Wilson's disease and in haemochromatosis.

In the present studies radio-opaque deposits in the substance of the intervertebral disc were shown to contain both calcium (alizarin red $S$ positively) and companion anions such as phosphate (von Kossa positively).

Green/white fluorescence after the application of the morin technique suggested the presence of pyrophosphate ${ }^{6}$ and this was further supported by the weak positive birefringence exhibited by many of the deposits. ${ }^{7}$

Dieppe et $\mathrm{l}^{8}$ have described the use of scanning electron microscopy (SEM) and $\boldsymbol{x}$-ray microanalysis in the distinction of the several forms of calcium phosphate (calcium hydroxyapatite, calcium pyrophosphate dihydrate, calcium orthophosphate dihydrate and dicalcium phosphate dihydrate) which may occur in articular and other tissues. Crocker $e t$ $a l^{9}$ have also described a correlative light microscopical and $x$-ray microanalytical method of identifying calcium pyrophosphate dihydrate crystals of differing morphology and calcium hydroxyapatite crystals in synovial fluid. These workers described a P:Ca ratio of $0.95 \pm 0.02$ obtained from pure triclinic calcium pyrophosphate dihydrate and a value of $0.45 \pm 0.02$ from pure calcium hydroxyapatite. The P:Ca value of 0.96 obtained from the crystalline deposits in the present case corresponds closely to that of pure triclinic CPPD and strongly suggests that the deposits are composed of CPPD. The value 
of 0.57 obtained from lamellar bone is higher than that of pure calcium hydroxyapatite. However, it is recognised that the $\mathrm{P}$ and $\mathrm{Ca}$ content of bone is not exclusively in the form of calcium hydroxyapatite. There is a proportion in the form of amorphous calcium phosphate which will increase the ratio.

The importance of using analytical techniques in determining the nature of the calcific deposits is emphasised by a recent report by Hoffman et al. ${ }^{10}$ They described a calcium oxalate microcrystallineassociated arthritis in end-stage renal disease. The crystals were rod-shaped, positively birefringent and bore electron-microscopical similarities to CPPD. However, $x$-ray microanalysis showed an absence of phosphate and allowed their true chemical nature to be pursued and elucidated.

The studies reported herein allow the confident identification of radio-opacities in the intervertebral discs of a case of Wilson's disease as deposits of CPPD. The peripheral joints in the case were not available for study and therefore it is not known if there was an associated peripheral chondrocalcinosis.

In some discs the calcific deposits were concentrated in the cartilaginous end-plates, where new trabecular bone formation was also noted. This resulted in irregularity and narrowing of the disc and sclerosis of the end-plates. It is tempting to speculate that the calcific deposits and the new bone formation are related with the former perhaps acting as an inductive influence for the latter.
We wish to thank Mrs Carmen Wickremasuriya for typing the manuscript and the Electron Optical Centre, University of Adelaide, for the use of their electron probe facilities.

\section{References}

' Hamilton EBD. Diseases associated with CPPD deposition disease. Arthritis Rheum 1976;19:355-7.

${ }^{2}$ Boudin G, Pepin B, Hubault A. Les arthropathies de la maladie de Wilson. Societe Medicale des Hopitaux de Paris 1963;114:617-22.

${ }^{3}$ Feller RE, Schaumacher HR. Osteoarticular changes in Wilson's disease. Arthritis Rheum 1972;15:259-66.

4 Delves HT. The clinical value of trace-metal measurements. Essays Med Biochem 1976;2:37-73.

${ }^{5}$ McCarty DJ, Pepe PF, Solomon SD, et al. Inhibition of human erythrocyte pyrophosphatase activity by calcium, cupric and ferrous ions. Arthritis Rheum 1971;13:336.

${ }^{6}$ Chaplin AJ. Calcium pyrophosphate. Histological characterization of crystals in pseudogout. Arch Pathol Lab Med 1976;100:12-15.

' Currey HLF. Examination of joint fluids for crystals. Proc $R$ Soc Med 1968;61:969-71.

${ }^{8}$ Dieppe PA, Huskisson EC, Crocker P, Willoughby DA. Apatite deposition disease. A new arthropathy. Lancet 1976;i:266-8.

${ }^{9}$ Crocker PR, Dieppe PA, Tyler G, Chapman SK, Willoughby DA. The identification of particulate matter in biological tissues and fluids. $J$ Pathol 1977;121:37-40.

${ }^{10}$ Hoffman GS, et al. Calcium oxalate microcrystalline-associated arthritis in end-stage renal disease. Ann Intern Med 1982;97:36-42.

Requests for reprints to: Dr J McClure, Department of Histopathology, UHSM, Nell Lane, Withington, Manchester M20 8LR. 